\title{
Mobile phone-based asthma self-management aid for adolescents (mASMAA): a feasibility study
}

\author{
This article was published in the following Dove Press journal: \\ Patient Preference and Adherence \\ 7 January 2014 \\ Number of times this article has been viewed
}

\author{
Hyekyun Rhee' \\ James Allen² \\ Jennifer Mammen' \\ Mary Swift ${ }^{2}$ \\ 'School of Nursing, ${ }^{2}$ Department \\ of Computer Science, University \\ of Rochester, Rochester, NY, USA
}

Correspondence: Hyekyun Rhee School of Nursing, University of Rochester, 60I Elmwood Avenue, Box SON, Rochester, NY I4642, USA

Tel +I 5852763775

Fax + I 585273 I270

Email hyekyun_rhee@urmc.rochester.edu
Purpose: Adolescents report high asthma-related morbidity that can be prevented by adequate self-management of the disease. Therefore, there is a need for a developmentally appropriate strategy to promote effective asthma self-management. Mobile phone-based technology is portable, commonly accessible, and well received by adolescents. The purpose of this study was to develop and evaluate the feasibility and acceptability of a comprehensive mobile phone-based asthma self-management aid for adolescents (mASMAA) that was designed to facilitate symptom monitoring, treatment adherence, and adolescent-parent partnership. The system used stateof-the-art natural language-understanding technology that allowed teens to use unconstrained English in their texts, and to self-initiate interactions with the system.

Materials and methods: mASMAA was developed based on an existing natural dialogue system that supports broad coverage of everyday natural conversation in English. Fifteen adolescent-parent dyads participated in a 2-week trial that involved adolescents' daily scheduled and unscheduled interactions with mASMAA and parents responding to daily reports on adolescents' asthma condition automatically generated by mASMAA. Subsequently, four focus groups were conducted to systematically obtain user feedback on the system. Frequency data on the daily usage of mASMAA over the 2-week period were tabulated, and content analysis was conducted for focus group interview data.

Results: Response rates for daily text messages were $81 \%-97 \%$ in adolescents. The average number of self-initiated messages to mASMAA was 19 per adolescent. Symptoms were the most common topic of teen-initiated messages. Participants concurred that use of mASMAA improved awareness of symptoms and triggers, promoted treatment adherence and sense of control, and facilitated adolescent-parent partnerships.

Conclusion: This study demonstrates the utility and user acceptability of mASMAA as a potential asthma self-management tool in a selective group of adolescents. Further research is needed to replicate the findings in a large group of adolescents from sociodemographically diverse backgrounds to validate the findings.

Keywords: asthma, self-management, text messaging, adolescents

\section{Introduction}

Asthma represents an increasingly serious health problem in young people in the US, with rapidly rising mortality and morbidity over the past 2 decades. ${ }^{1}$ Asthma morbidity in pediatric populations has been inadequately addressed, despite ongoing advances in treatment..$^{2-5}$ Nearly $12 \%$ of high school students in the US reported current asthma diagnosis in 2011.6 Adolescents are at particularly high risk for asthma morbidity, ${ }^{7-9}$ due in part to the transitional nature of their development that poses serious behavioral and social threats to optimum asthma management. ${ }^{10-14}$ Parental involvement and 
support can potentially counterbalance suboptimal asthma care, and is critical in achieving optimum asthma control in adolescents. ${ }^{15-18}$ Mobilizing and strengthening adolescentparent partnerships in enhancing efforts for asthma care in adolescents is therefore critical. Nonetheless, parents' capacity to effectively assist adolescents' asthma management is often compromised due to sparse or ineffective communications between adolescents and parents, resulting in limited information sharing. Thus, there is a need for an innovative approach that facilitates effective adolescent-parent partnerships as part of asthma self-management in adolescents.

Mobile phones have become an important method of adolescent communication. According to the Pew Research Center, ${ }^{19} 77 \%$ of adolescents aged $12-17$ years own a mobile phone; this rate is even higher $(87 \%)$ in older adolescents aged 14-17 years. Text messaging is a dominant daily mode of communication. Sixty-three percent of all adolescents reported that they had exchanged text messages every day with people in their lives, and the median number of texts sent on a typical day was 60 in 2011 . On average, adolescents spend 95 minutes a day sending and receiving texts. ${ }^{20}$ Given widespread ownership, high-volume daily use, particular appeal to adolescents, and adaptability in features, mobile phones can be used as a potentially powerful tool in aiding adolescents' asthma self-management and facilitating parent partnerships.

Mobile phone-based technologies have been used to facilitate self-management of various health conditions, with evidence of efficacy in improving health outcomes and patients' self-management capacity, primarily medication adherence. ${ }^{21,22}$ Recent studies have reported the successful application of interactive mobile phone-based systems in the daily assessment of symptoms and medication adherence in adolescents with asthma. ${ }^{23,24}$ Particularly, text messaging has demonstrated utility for asthma self-management, as it is readily accessible, location-independent, and facilitates interaction with other users. ${ }^{25-27}$ Nonetheless, research on use of texting services to promote asthma self-management in adolescents is limited, despite its particular value in health promotion during adolescence. ${ }^{28}$ To date, only two studies have reported the application of text messaging in designing interventions to improve medication adherence in adolescents using reminder texting ${ }^{29}$ and self-motivational texting for medication adherence. ${ }^{30}$ The use of text messaging as a comprehensive self-management aid to facilitate medication adherence, asthma monitoring, and parent-teen partnerships has yet to be researched. Therefore, the purpose of this study was to develop a comprehensive mobile phone-based asthma self-management aid for adolescents (mASMAA), primarily using the texting feature, and to demonstrate the feasibility and user acceptability of mASMAA through the analysis of usage data for 2 weeks and quantitative and qualitative user feedback. In this study, we defined feasibility as the extent to which adolescents and parents successfully used the multiple features of mASMAA as intended for a consecutive 2 weeks, and the degree of convenience and usefulness of mASMAA perceived by the users.

\section{Materials and methods Study-design overview}

The study comprised two phases: 1) design and development of mASMAA and 2) pilot testing the prototype mASMAA for feasibility and user acceptability.

\section{mASMAA-development phase}

mASMAA is an interactive system that primarily uses a text-messaging modality as a means of interaction with users. It is an extension of the existing TRIPS (The Rochester Interactive Planning System) natural dialogue system with an extensive lexicon of conversational English. ${ }^{31-36}$ We modified and expanded the TRIPS existing lexicon and ontology by adding common vocabularies and expressions specific to asthma (eg, coughing, wheezing), common asthma triggers (eg, pollen, smoke, pets), and medication types (eg, rescue and control medications). Additionally, common texting abbreviations and expressions were added (eg, "18r" for later, "ur" for your, and "thx" for thanks). We also developed a new dialogue manager that enabled the interpretation of patient reports based on their personal medication schedules, the generation of questions and reminders to help the teen monitor symptoms and manage their medications and activity. The natural dialogue system can afford user experience that resembles real-life as closely as possible by allowing users to exchange text messages as if they were with a real person. Natural language processing was especially key to interpreting user-initiated texts, a capability that could not be easily achieved using forms. The goal was to make the system more appealing to the teens, thus eliciting greater and longer interest in and use of the system.

mASMAA was designed with several unique features. First, it was customizable, and could be tailored based on a user's daily routines, specific medications, and medication schedules. The automated daily asthma-monitoring inquiries and medication reminders could be sent according to user-defined preferences. Second, the system, using its natural language-processing technology, was able to 
process and respond to user-initiated text messages related to asthma at any time, as well as understand more complex and nuanced answers to system inquiries (eg, the answer "Only when I played baseball" to a question about whether they had asthma symptoms today). Third, automated monitoring of all text messages allowed for tracking of asthma conditions, including symptoms, activity level, and use of rescue and control medications, as indicated by users. A daily report summarizing this information was sent to parents via email. Fourth, the program utilized a "wizard" interface, a web page that enables a person ("wizard") to monitor interactions between mASMAA and a user and intervene whenever necessary. Texting alerts were automatically sent to the wizard whenever messages could not be handled due to unusual or complex information. The wizard, a certified asthma educator, would then temporarily assume control to reciprocate with an appropriate response to the user.

\section{mASMAA pilot-testing phase}

\section{Setting and sample}

Potential adolescent-parent dyads were recruited from clinical settings, including the emergency department and primary care clinics in a university medical center using clinic referrals or study flyers. Eligibility criteria for adolescent participants included 1 ) asthma diagnosis ( $\geq 1$ year) confirmed by medical records, 2) aged 13-17 years, 3) use of health care service for asthma in the past 12 months, and 4) texting-capable mobile phone ownership. A biological or adoptive parent or legal guardian who resided in the primary residence of the eligible adolescent was invited to participate in the study. Capacity to speak and read English was required to be eligible for both parents and adolescents.

\section{Study procedure}

Enrolled adolescent-parent dyads participated in the 2-week trial of the prototype mASMAA. At enrollment, participants completed a demographic form, and the Asthma Control Test provided the names of current asthma medications and indicated the preferred time for receiving reminder texts from mASMAA. During the trial, adolescents responded to six routine asthma-diary questions (two in the morning and four at bedtime) and control medication reminders sent daily by mASMAA. Adolescents' responses were unstructured, as no predetermined set of responses was provided. Text messages were sent at a time determined by each of the adolescents based on his or her preference and medication schedule. Interactions were individualized to reflect participants' current medications and frequency of administration (eg, once per day or twice per day). In addition, adolescents were encouraged to text self-initiated messages related to asthma (eg, symptoms, medications, activity) at least twice per day. Medication reminders were not sent if the adolescent had already self-initiated a report that the medication had been taken. Figure 1 is a sample interaction log recording simple interactions between the system and a participant. The majority of participants' responses were successfully processed and responded to by mASMAA, while "atypical" or unexpected responses that could not be handled automatically

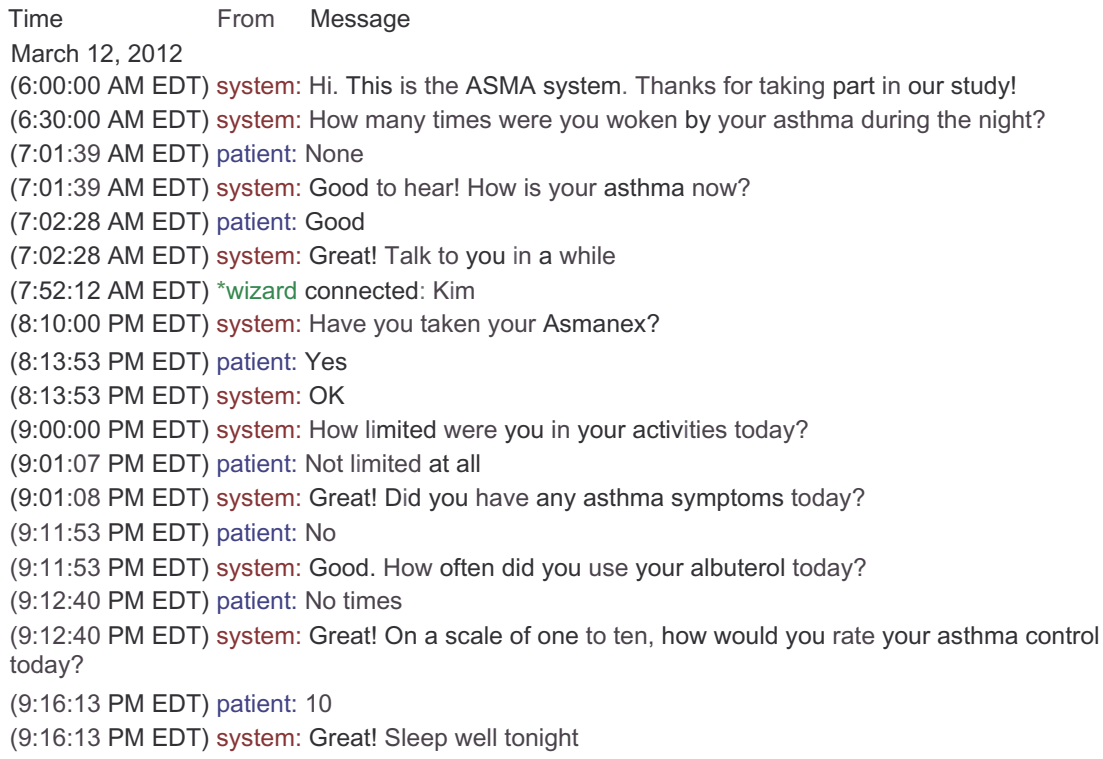

Figure I A sample web-based interaction log recording interactions between the mobile phone-based asthma self-management aid for adolescents and a participant. Abbreviations: EDT, eastern daylight time; ASMA, asthma self-management aid. 
were managed and responded to by the wizard. Below is an example interaction between a teen and the wizard.

Teen: Bad morning cough, chest hurts, needing my ProAir this morning.

Wizard: Did taking ProAir help? How are you feeling now?

Teen: OK, ProAir helping a little, had to rest.

Wizard: Make sure that your parents know your symptoms and you took ProAir.

Teen: OK, starting to feel a little better.

Based on the information collected throughout the day, the system generated and sent daily asthma reports to parents via email. The daily reports informed the parents of their adolescents' asthma-control levels (well controlled, somewhat controlled, or poorly controlled), levels of activity (sedentary, somewhat active, or very active), frequency of rescue medication (eg, albuterol) use, and control-medication use. Upon receipt of the daily report, parents were asked to send brief confirmation emails to the study team.

On completion of the 2-week trial, four focus groups were held: two adolescent-only groups and two parent-only groups. Focus groups were conducted using semistructured questions to systematically capture users' experience with mASMAA. Main categories assessed included user-friendliness (how easy and convenient to use), perceived benefits of mASMAA, and suggestions for improvement. Focus group lengths were between 45 and 60 minutes. All interviews were audio-recorded and transcribed verbatim. Identifying names and health identifiers were removed. The Institutional Review Board approved the study protocol, and participating parents and adolescents provided informed consent and assent, respectively.

\section{Data analysis}

Average response rate for each of six routine asthma-monitoring questions was calculated. Self-initiated text messages were sorted based on content (symptom-, medication-, and activity-related). Three researchers conducted the content analysis of the interview transcripts independently, and codes were compared for similarities and differences. Any discrepancies in coding were discussed and reconciled. The qualitative analysis software Atlas.ti v7 (Atlas.ti Scientific Software Development, Berlin, Germany) was used to facilitate content analysis.

\section{Results}

\section{Sample characteristics}

Of the 20 adolescent-parent dyads originally planned, 16 adolescent-parent dyads were enrolled in the study during a brief 6-month recruitment stage. We approached
25 adolescents who met the eligibility criteria for recruitment, of whom twelve were enrolled (48\% enrollment rate). An additional four dyads initiated contact in response to the study flyer and subsequently enrolled in the study. Of 16 enrolled dyads, one dyad (a flyer responder) was excluded from the analysis due to their unsuccessful attempt to initiate the trial. Table 1 summarizes the demographic characteristics of the sample. Mothers represented the majority $(80 \%, n=12)$ of participating parents. On average, adolescent participants had lived with asthma for 10.8 years (range 3-16 years, standard deviation 4.0). Almost all adolescent participants $(87 \%, n=13)$ were on daily control medications. Eighty percent of the sample $(n=12)$ perceived that their asthma was well or completely controlled in the past 4 weeks. However, according to the Asthma Control Test assessing asthma symptoms, activity limitations, and use of rescue medications, $75 \%(n=9)$ of those reporting wellcontrolled asthma were found to have poorly controlled asthma, with 38\% reporting nighttime symptoms, 38\% reporting daytime symptoms, 56\% reporting activity limitation due to asthma, and 44\% using short-term relief medication more than 2 days per week.

\section{Summary of mASMAA usage}

The average response rates to each diary question ranged from $81 \%$ to $97 \%$ (Table 2). In general, response rates for the questions sent in the morning were higher than those for bedtime questions. Based on the responses to

Table I Demographic and asthma-related characteristics of the study sample

\begin{tabular}{ll}
\hline & $\mathbf{n}(\%)$ \\
\hline Age, years, mean, (SD) & $15(\mathrm{I} .5)$ \\
Sex & \\
Male & $9(60 \%)$ \\
Female & $6(40 \%)$ \\
Race & \\
White & $8(53 \%)$ \\
African American/black & $6(40 \%)$ \\
Asian & $1(7 \%)$ \\
Annual household income & \\
$<\$ 10,000$ & $4(27 \%)$ \\
$\$ 10,000-\$ 30,000$ & $1(6 \%)$ \\
$\$ 30,000-\$ 50,000$ & $1(6 \%)$ \\
$\$ 50,000-\$ 70,000$ & $1(6 \%)$ \\
$>\$ 70,000$ & $7(47 \%)$ \\
Missing & $1(6 \%)$ \\
Years with asthma diagnosis, mean (SD) & $10.8(4)$ \\
Control medication & $13(87 \%)$ \\
Not well controlled or poorly controlled asthma & $12(80 \%)$ \\
\hline
\end{tabular}

Abbreviation: SD, standard deviation. 
Table 2 Adolescents' response to the mobile phone-based asthma self-management aid for adolescents' daily asthmamonitoring questions (standard questions)

\begin{tabular}{|c|c|}
\hline Questions & $\begin{array}{l}\text { Response } \\
\text { rates }^{\mathrm{a}}\end{array}$ \\
\hline \multicolumn{2}{|l|}{ Morning questions } \\
\hline $\begin{array}{l}\text { I. How often were you woken by your asthma } \\
\text { during the night? }\end{array}$ & $97 \%$ \\
\hline $\begin{array}{l}\text { 2. How bad were your asthma symptoms when } \\
\text { you woke up this morning? }\end{array}$ & $90 \%$ \\
\hline \multicolumn{2}{|l|}{ Bedtime questions } \\
\hline $\begin{array}{l}\text { 3. How limited were you in your activities today } \\
\text { because of your asthma? }\end{array}$ & $89 \%$ \\
\hline $\begin{array}{l}\text { 4. How much did you have asthma symptoms (coughing, } \\
\text { wheezing and/or shortness of breath) today? }\end{array}$ & $86 \%$ \\
\hline $\begin{array}{l}\text { 5. How often did you use your short-term } \\
\text { bronchodilator ("quick-relief" "rescue" medicine } \\
\text { like Ventolin/Proventil/albuterol) today? }\end{array}$ & $84 \%$ \\
\hline 6. How would you rate your asthma control today overall? & $81 \%$ \\
\hline
\end{tabular}

Notes: ${ }^{\text {TT }}$ The percentage of the time a participant responded for each time the questions were asked over the 2 -week period. The rate was the average of all participants' response rates.

day- and bedtime symptoms, activity limitations, and rescue medication use, $60 \%$ of the sample were found to experience uncontrolled asthma for 2 days or more during the course of the 2-week trial.

Each adolescent on average submitted 19 self-initiated text messages (range 3-38) to mASMAA during the trial period. When the self-initiated messages were sorted based on content, symptom related messages were most common (69\%), followed by activity-related (48\%) and medication-related $(10 \%)$ messages. Twenty-nine percent of the self-initiated messages included two or more of these categories.

Parents received daily reports from mASMAA via email summarizing their adolescents' asthma condition. The parents sent confirmation emails to the study team acknowledging their receipt. The average number of confirmation emails per parent was twelve (range 7-14) throughout the 2 -week trial period, indicating high parental involvement.

\section{Summary of focus group interviews}

Of 15 adolescent-parent dyads, ten dyads participated in four focus groups (two for adolescents and two for parents). Each group was attended by five participants. Eighty percent $(n=8)$ of adolescent participants were males, and 40\% were African American. Mothers represented 80\% $(n=8)$ of parent participants. No significant differences in age, household income, or asthma-control scores were found between focus group attendees and those who did not attend.

\section{mASMAA feasibility and acceptability}

Overall experience with mASMAA was favorable for both parents and adolescents. Almost all adolescent and parent participants concurred that mASMAA was easy and convenient. Adolescents particularly favored mASMAA because it was "new" and interactive ("You send a message and it would give something back"), and using their own phone ("I always have my phone, my phone is - this is my life"). Adolescents were able and willing to make adjustment to their routines to accommodate mASMAA and became accustomed to interactions with mASMAA easily ("You get used to it and it becomes routine"; "I feel like it becomes normal, just like ... an instinct to do it"). For most participants, the 2-week trial was "smooth," although a few technical issues were reported associated with initial login. Parents felt that mASMAA was developmentally appropriate and appealing, given adolescents' general attraction to technology ("He's into all of that new technology"), ubiquitous use of texting, and the system's potential appeal to foster independence in managing asthma ("As the kids get older, I think ... the teens just want to own and control the thing").

Parents commented that the daily parent reports were informative and practical, and provided adequate amounts of information. Most adolescents and parents concurred that mASMAA could be a useful and convenient tool for adolescents' asthma self-management, and believed that other adolescents with asthma and their parents would like to use the system.

\section{Potential benefits of mASMAA}

Participants identified several benefits of mASMAA in improving asthma self-management.

\section{Increased awareness of symptoms and triggers}

All adolescents concurred that regular use of mASMAA had helped them become more aware and "keep track of symptoms." An adolescent stated:

You could like share like [...] your symptoms with the machine or whatever and it helped you realize what your symptoms were - actually were. It's [...] sometimes you don't really um [...] like [...] realize that you've had symptoms - you actually have to think about it. [Adolescent]

The recognition of the symptoms often made adolescents realize and be attentive to symptom triggers, as indicated in the following statement. 
I would be like, 'Oh, I never noticed how much I will cough and wheeze.' Like I have a dog, and when I get around her I would cough and wheeze and stuff. So I never really noticed it until I - I would get the text messages - how bad my symptoms were really. 'Cause I was always on the go, so I never paid any attention to my symptoms. [Adolescent]

Daily reports from mASMAA also increased parents' awareness of their adolescents' asthma condition and triggers. All parents in a focus group stated that they felt approximately $50 \%-75 \%$ more aware of their adolescents' asthma condition.

It did help me know that how he does like even with after school sports. Like how much he needs an inhaler 'cause sometimes he doesn't tell me like 'Oh, I have it' because he thinks I'm gonna like, 'Oh, you can't play sports,' but yeah, it really was good for me to know like how many attacks or how he felt. [Parent]

\section{Improved asthma self-management} and medication adherence

Both adolescent and parent groups reported that regular use of mASMAA had made a positive impact on asthma selfmanagement. Adolescents stated, "It [mASMAA] helped me remember and manage my asthma." Increased awareness of symptoms and triggers allowed them to be more effective and proactive in managing asthma.

I feel like it could help you manage your asthma because it like tells you when like you're having flare-ups and what time of day and then that can help you find out why because you can reflect and think back what you were doing then or what you were exposed to. [Adolescent]

Knowing that there is a system in place to assist your children is a wonderful thing to try to be more proactive rather than reactive. [Parent]

Specifically, adolescents and parents appreciated medication reminders from mASMAA. Many adolescents admitted that they often forgot to take control medications, and the texting reminders helped in not only remembering but also establishing their routines to make it easier to remember (eg, "Get in to a scheduled routine"; "Helped regulate when I took my medicine").

It [mASMAA] reminded me when to take my Flovent, because I used to always forget to take it in the morning, and it helped me keep myself on track. Now I - even after the program's over with I'm still doing it. [Adolescent]
I just thought that it really helped to foster more compliance with him. Knowing that those texts were going to be coming in [...] um, obviously made him more selfdirected in terms of making sure he took his meds as needed. [Parent]

\section{Improved sense of control over asthma} and its management

Three parents shared that the system helped their teens foster a sense of control over asthma and confidence in managing asthma based on their observation during the trial. A parent stated:

I found was that my son felt he had control. Which some - often times with a chronic disease you feel like you lose that control [...] He liked that he was - he felt he was in charge of that. And I really like that because we're trying to really [...] help him self-manage his asthma. And so I really liked with him doing this [mASMAA trial] he really liked it that he was in control of his own disease. [Parent]

As parents noted, "Adolescents want to own and control the thing," and believed that "The fact that the text came in and asked versus mom saying it every day, 'Did you do this?' was better for them." Therefore, the improved sense of control is a developmentally important benefit that is essential to behavioral changes for adequate asthma self-management. In fact, adolescents reported increased independence during the trial, as indicated in their improved self-management (eg, taking medications) without parents' prompting.

\section{Accessibility of asthma-related advice}

Six adolescents noted that easy and convenient access to advice related to asthma symptoms and management anywhere and anytime through mASMAA was helpful.

I think it would help them just 'cause if they needed someone's opinion or they didn't know what to do they can text you and they'd send back a quick answer. [Adolescent]

Parents were concerned that they would not always be available to talk and have expertise to help their adolescents with asthma-related issues. Therefore, they viewed that having a technology such as mASMAA that could offer "the second option of communication" and provide timely and professional advice on asthma management would be an important benefit. 
Improved adolescent-parent communication

Parents viewed that daily reports from mASMAA were invaluable as "a flashback" or "an overview" of their teen's asthma. Parents acknowledged that the increased awareness of their adolescent's asthma symptoms and medication adherence could lead to improved communication, as indicated in the following statement.

I liked that report. It gave me that report. At the end of the day, I would look at my email and I would see like, in the morning you know xxx said this is an incident, and so I would go to him and be like, oh, what happened? [Parent]

Furthermore, parents concurred that the increased awareness and communication also helped parents to be more effective in facilitating and assisting teens' self-management.

The amount of sharing information sometimes is limited. And it's not because they do it on purpose, they're just busy. You know? They're busy in their lives. And for me, the emails coming in to say, 'Hey, you know your son had - you know, some asthma problems today,' and I would have normally not known that [...] was very very helpful $[\ldots]$ for me. Because then I started really looking at, OK, what's [...] what are the patterns? What's going on? Have you taken your meds? Is there something else that might be triggering it? So it just kind of helped me problem-solve what was going on and [...] get him involved in the conversation piece so that I could try to educate him on possible triggers that were, um, causing his breakthrough symptoms. [Parent]

None of the adolescents discussed the improved communication with parents as a benefit.

\section{Overall focus group summary}

Adolescents and parents agreed that the benefits of mASMAA could contribute to effective asthma self-management. Three parents attributed their adolescents' improved asthma control during the trial period to mASMAA. Five parents believed that the system could be more beneficial and helpful for those whose asthma is not controlled.

Negative experiences with mASMAA were also discussed. Most of these occurred at the beginning of the trial, due primarily to technical challenges, such as difficulties in establishing the initial connection with the system, which was often the case when participants used prepaid phone services. Participants also reported challenges associated with the system's limited lexicon dictionary that often triggered the system's automatic response of "I don't understand," requiring the wizard's intervention. Participants made suggestions for the improvement of the system, including expanding mASMAA's language-recognition capacity and maximizing flexibility by allowing users to control the timing of interactions with the system. Parents believed that mASMAA's features to facilitate information sharing with health care providers could further strengthen its clinical usefulness.

\section{Discussion}

Despite a growing literature on the application of text messaging in addressing self-care needs in a variety of chronic illnesses, studies systematically evaluating user acceptance of such applications are limited. ${ }^{22}$ This study attempted to address the gap by using primarily a qualitative approach in evaluating a texting-based system - mASMAA. mASMAA is a complex and comprehensive self-management system that facilitates daily asthma monitoring and parent-adolescent partnerships, as well as medication adherence.

This study demonstrated the feasibility of using an automated text-messaging system that can interpret and manage conversational English text messages in monitoring asthma symptoms daily. This method for symptom monitoring was well received by adolescents, who acknowledged increased awareness of symptoms and triggers as well as ability to keep track of symptoms. The use of text messaging as an asthmamonitoring method has been demonstrated to have feasibility in and acceptability by adult patients, ${ }^{25,37}$ and has been shown to improve adult users' awareness, knowledge, and self-efficacy in monitoring and managing symptoms. ${ }^{25-27,38}$ Although symptom monitoring has been emphasized as a cornerstone for optimum asthma management, ${ }^{39,40}$ adolescents' adherence to daily monitoring is poor and inconsistent. ${ }^{2,40,41}$ In this study, $60 \%$ of the sample inaccurately perceived that their asthma was well controlled when the levels of symptoms indicated otherwise. Children's tendency to overestimate their asthma control despite ongoing symptoms has been reported in a large clinical-based study. ${ }^{42}$ The poor perception of asthma control further underscores the need for an innovative system to promote symptom monitoring and to provide adequate and timely feedback. This study suggests text messaging as an alternative method for daily symptom monitoring in adolescents.

Text messaging has been used as a medication reminder for a variety of health conditions, including human immunodeficiency virus infection, ${ }^{43}$ diabetes, ${ }^{44,45}$ and asthma. ${ }^{29,30}$ Many studies have shown improved medication adherence and user satisfaction with the texting reminders. ${ }^{25,37,46-48}$ Consistent with previous findings, the tailored medication 
reminders delivered by mASMAA were well received by adolescents and parents. Our focus group data indicate that adolescents were not simply passive recipients of reminders. Instead, they actively used the reminders to establish and schedule daily routines of asthma self-management, contributing to an increased sense of control over disease and its management. mASMAA's capacity to foster a sense of control appeals to users' developmental needs for independence and autonomy. Although this study did not directly measure medication adherence, it is noteworthy that participants attributed their improved treatment adherence and asthma control during the study period to the reminders from mASMAA. Future research is needed to evaluate adequately the impact of mASMAA's medication reminders on treatment adherence and asthma control.

Participants, particularly adolescents, appreciated access to professional asthma-related advice transcending time and space afforded by mASMAA. Teen-initiated text messages to mASMAA predominantly concerning symptoms may reflect the adolescents' needs for information and advice in dealing with asthma symptoms. In that sense, mASMAA's capacity to address adolescents' asthma-related concerns and questions in a professional and timely manner can be an invaluable asset. mASMAA adopted a "wizard" concept to sort and respond to messages that could not be handled automatically. The wizard, an actual person, responded to many teen-initiated messages. Therefore, adolescents inevitably experienced some delays, no longer than an hour, in receiving feedback after their self-initiated texting, depending on the wizard's availability at that moment. Further developed mASMAA could shorten the waiting time by enhancing its capacity to automate feedback based on the concrete dialogue samples between the wizard and users obtained in this study. Maximizing the system's capacity to automate its interactions could potentially contribute to system-operation cost savings by reducing costly and time-consuming human interventions. Nonetheless, our study participants appear to favor mASMAA's ability to allow a person to intervene, in case the nature of the issue transmitted by a teen warrants immediate medical attention.

mASMAA is unique and innovative in its capacity to incorporate a parent component seamlessly in its daily operation by providing parental reports summarizing the adolescent's reported asthma conditions each day. Parents' high response rates to daily reports and positive perceptions about the reports indicate not only parents' receptiveness but also the system's potential value in eliciting effective parent partnerships in asthma management. Parent partnerships have been recognized as an important part of asthma management in adolescents. ${ }^{18,49-51}$ As indicated in parent focus groups, however, building teen-parent partnerships can be elusive due to sparse or counterproductive interactions between the two parties, thus presenting challenges for parents to obtain necessary information from teens to provide timely and adequate assistance. mASMAA demonstrated its capacity potentially to address communication barriers. Further study is necessary to quantify changes in parental participation in teens' daily asthma management due to mASMAA. Parents made a few suggestions to make this feature more practical and clinically useful, such as daily reports sent via text messages as well as emails, an instant alert feature informing parents of teens' asthma conditions warranting immediate assistance, and capability to archive daily reports to be shared with health care providers. Incorporating these enhanced features in mASMAA would increase its accessibility and clinical relevance.

Overall, the study demonstrated the feasibility of mASMAA as a daily tool for asthma self-management in adolescents. Nonetheless, cautions are warranted in interpreting findings. This study was not intended to examine the effects of mASMAA on actual management behaviors or asthma outcomes (eg, asthma control, quality of life), although some participants reported changes in such behaviors as avoiding triggers, monitoring symptoms, and adhering to preventive measures in connection with mASMAA use. The brief 2-week evaluation period also prevented us from speculating on any long-term effects or sustainability of the system. In addition, given the small number of participants and overrepresentation of boys in focus groups, user perceptions and opinions may not be representative of a wide range of potential adolescent and parent users. This study did not collect quantitative data offering validation of participants' perceptions about the system's benefits. Further research is needed to quantify the perceived benefits using psychometric and physiological measures. Using emails to deliver daily parent reports may limit the user base of mASMAA, as it would discriminate against those who cannot afford a computer and the Internet subscription. Being at the prototype beta-testing stage, technical challenges were anticipated and encountered, causing frustration in some participants. Further refinement of the system based on a detailed automated asthma-management algorithm is warranted.

\section{Conclusion}

The majority of adolescents and parents agreed that mASMAA was an attractive and convenient option to facilitate asthma self-management, having acknowledged the mul- 
tiple benefits in promoting a sense of control, awareness of symptoms/triggers, treatment adherence, and adolescentparent partnerships. Some participants even noted an improved asthma condition during the study period. However, quantification of the positive effects of mASMAA and longterm sustainability of the system and its impact on asthma outcomes remain to be determined. In order to enhance user acceptance and clinical usefulness, mASMAA needs to overcome technical challenges and shortcomings (eg, incompatibility with prepaid phones, limited lexicon) and expand its functionality by incorporating user suggestions offered in the study. In addition, platforms for sharing the daily reports with parents need to be broadened beyond the email format to allow the system to be accessible to the families of a wide range of socioeconomic backgrounds. Given mobile phones' widespread ownership even among those of low socioeconomic status, ${ }^{52}$ mASMAA could provide practical and low-cost asthma self-care assistance, particularly to inner-city adolescents, who are disproportionately affected by asthma morbidity, yet have limited access to personal and financial resources needed for adequate self-management.

\section{Acknowledgments}

This study was supported by a Provost Multidisciplinary Award from the University of Rochester. We are grateful to Ms Kimberly Comeau for coordinating the study from subject recruitment to data analysis and serving as wizard for the trial. We also thank Mr William de Beaumont for providing his technical expertise in overseeing the daily operation of mASMAA. The principal investigators (Rhee and Allen) have full access to all the data in the study, and take responsibility for the integrity of the data and the accuracy of the data analysis.

\section{Disclosure}

The authors report no conflicts of interest in this work.

\section{References}

1. Akinbami LJ, Moorman JE, Liu X. Asthma prevalence, health care use, and mortality: United States, 2005-2009. Natl Health Stat Report. 2011;(32):1-14.

2. Fuhlbrigge AL, Guilbert T, Spahn J, Peden D, Davis K. The influence of variation in type and pattern of symptoms on assessment in pediatric asthma. Pediatrics. 2006;118(2):619-625.

3. Adams RJ, Fuhlbrigge A, Guilbert T, Lozano P, Martinez F. Inadequate use of asthma medication in the united states: results of the asthma in America national population survey. J Allergy Clin Immunol. 2002;110(1):58-64.

4. Bloomberg GR, Banister C, Sterkel R, et al. Socioeconomic, family, and pediatric practice factors that affect level of asthma control. Pediatrics. 2009;123(3):829-835.

5. Halterman JS, Aligne CA, Auinger P, McBride JT, Szilagyi PG. Inadequate therapy for asthma among children in the United States. Pediatrics. 2000;105(1 Pt 3):272-276.
6. Centers for Disease Control and Prevention. Asthma data and statistics. Available from: http://www.cdc.gov/healthyyouth/asthma/data.htm. Accessed November 21, 2013.

7. Brener ND, Kann L, Garcia D, et al. Youth risk behavior surveillance - selected steps communities, 2005. MMWR Surveill Summ. 2007;56(2):1-16.

8. Dey AN, Schiller JS, Tai DA. Summary health statistics for US children: National Health Interview Survey, 2002. Vital Health Stat 10. 2004;(221):1-78.

9. Akinbami LJ, Moorman JE, Garbe PL, Sondik EJ. Status of childhood asthma in the United States, 1980-2007. Pediatrics. 2009; 123 Suppl 3:S131-S145.

10. Price J, Kemp J. The problems of treating adolescent asthma: what are the alternatives to inhaled therapy? Respir Med. 1999;93(10): 677-684.

11. Dinwiddie R, Müller WG. Adolescent treatment compliance in asthma. J R Soc Med. 2002;95(2):68-71.

12. Zebracki K, Drotar D. Outcome expectancy and self-efficacy in adolescent asthma self-management. Child Health Care. 2004;33(2): 133-149.

13. McQuaid EL, Kopel SJ, Klein RB, Fritz GK. Medication adherence in pediatric asthma: reasoning, responsibility, and behavior. J Pediatr Psychol. 2003;28(5):323-333.

14. Fiese BH, Everhart RS. Medical adherence and childhood chronic illness: family daily management skills and emotional climate as emerging contributors. Curr Opin Pediatr. 2006;18(5):551-557.

15. Price JF. Issues in adolescent asthma: what are the needs? Thorax. 1996;51 Suppl 1:S13-S17.

16. Kyngas HA. Compliance of adolescents with asthma. Nurs Health Sci. 1999;1(3):195-202.

17. Orrell-Valente JK, Jarlsberg LG, Hill LG, Cabana MD. At what age do children start taking daily asthma medicines on their own? Pediatrics. 2008;122(6):e1186-e1192.

18. Penza-Clyve SM, Mansell CB, McQuaid EL. Why don't children take their asthma medications? A qualitative analysis of children's perspectives on adherence. J Asthma. 2004;41(2):189-197.

19. Pew Research Center. Teens and technology 2013. 2013. Available from: http://www.pewinternet.org/ /media/Files/Reports/2013/ PIP_TeensandTechnology2013.pdf. Accessed May 8, 2013.

20. Kaiser Family Foundation. Daily media use among children and teens up dramatically from five years ago. 2010. Available from: http:// kff.org/disparities-policy/press-release/daily-media-use-amongchildren-and-teens-up-dramatically-from-five-years-ago. Accessed November 4, 2013.

21. Krishna S, Boren SA, Balas EA. Healthcare via cell phones: a systematic review. Telemed J E Health. 2009;15(3):231-240.

22. de Jongh T, Gurol-Urganci I, Vodopivec-Jamsek V, Car J, Atun R. Mobile phone messaging for facilitating self-management of long-term illnesses. Cochrane Database Syst Rev. 2012;12:CD007459.

23. Mulvaney SA, Ho YX, Cala CM, et al. Assessing adolescent asthma symptoms and adherence using mobile phones. J Med Internet Res. 2013;15(7):e141.

24. Ryan D, Price D, Musgrave SD, et al. Clinical and cost effectiveness of mobile phone supported self monitoring of asthma: multicentre randomised controlled trial. BMJ. 2012;344:e1756.

25. Holtz B, Whitten P. Managing asthma with mobile phones: a feasibility study. Telemed J E Health. 2009;15(9):907-909.

26. Ryan D, Cobern W, Wheeler J, Price D, Tarassenko L. Mobile phone technology in the management of asthma. J Telemed Telecare. 2005;11 Suppl 1:43-46.

27. Ostojic V, Cvoriscec B, Ostojic SB, Reznikoff M, Stipic-Markovic A, Tudjman Z. Improving asthma control through telemedicine: a study of short-message service. Telemed J E Health. 2005;11(1):28-35.

28. Pinnock H, Slack R, Pagliar C, Price D, Sheikh A. Understanding the potential role of mobile phone-based monitoring on asthma self-management: qualitative study. Clin Exp Allergy. 2007;37(5): 794-802. 
29. Britto MT, Munafo JK, Schoettker PJ, Vockell AB, Wimberg JA, Yi MS. Pilot and feasibility test of adolescent-controlled text messaging reminders. Clin Pediatr (Phila). 2012;51(2):114-121.

30. Seid M, D'Amico EJ, Varni JW, et al. The in vivo adherence intervention for at risk adolescents with asthma: report of a randomized pilot trial. J Pediatr Psychol. 2012;37(4):390-403.

31. Allen J, Byron D, Dzikovska M, Ferguson M, Galescu L, Stent A. An architecture for a generic dialogue shell. Nat Lang Eng. 2000;6(3-4):213-228.

32. Allen JF, Byron DK, Dzikovska M, Ferguson M, Galescu L, Stent A. Towards conversational human-computer interaction. $A I$ Mag. 2001;22(4):27-35.

33. Allen J, Ferguson G, Blaylock N, et al. Chester: towards a personal medical advisor. J Biomed inform. 2006;39(5):500-513.

34. Allen J, Swift M, de Beaumont W. Deep semantic analysis of text. In: Bos J, Delmonte R, editors. Semantics in Text Processing: STEP 2008 Conference Proceedings (Research in Computational Semantics). London: College Publications; 2008.

35. Ferguson G, Allen J, et al. CARDIAC: an intelligent conversational assistant for chronic heart failure patient health monitoring. 2009. Available from: http://www.aaai.org/ocs/index.php/FSS/FSS09/paper/ download/915/1175. Accessed November 4, 2013.

36. Ferguson G, Quinn J, Horwitz C, Swift M, Allen J, Galescu L. Towards a personal health management assistant. Biomed Inform. 2010;43(Suppl 5):S13-S16.

37. Prabhakaran L, Chee WY, Chua KC, Abisheganaden J, Wong WM. The use of text messaging to improve asthma control: a pilot study using the mobile phone short messaging service (SMS). J Telemed Telecare. 2010;16(5):286-290.

38. Lin S, Yang H. Exploring key factors in the choice of e-health using an asthma care mobile service model. Telemed E Health. 2009;15(9):884-890.

39. National Asthma Education and Prevention Program, Third Expert Panel on the Diagnosis and Management of Asthma. Expert Panel Report 3: Guidelines for the Diagnosis and Management of Asthma. Bethesda (MD): National Heart, Lung, and Blood Institute; 2007.

40. Bhogal S, Zemek R, Ducharme FM. Written action plans for asthma in children. Cochrane Database Syst Rev. 2006;(3):CD005306.

41. Rhee H, Belyea MJ, Elward KS. Patterns of asthma control perception in adolescents: Associations with psychosocial functioning. J Asthma. 2008;45(7):600-606.
42. Price D, Ryan D, Pearce N, Bawden R, Freeman D, Thomas M. The burden of paediatric asthma is higher than health professionals think: results from the asthma in real life (AIR) study. Prim Care Respir J. 2002;11(2):30-33.

43. Horvath T, Azman H, Kennedy GE, Rutherford GW. Mobile phone text messaging for promoting adherence to antiretroviral therapy in patients with HIV infection. Cochrane Database Syst Rev. 2012;3:CD009756.

44. Hanauer DA, Wentzell K, Laffel N, Laffel LM. Computerized automated reminder diabetes system (CARDS): e-mail and SMS cell phone text messaging reminders to support diabetes management. Diabetes Technol Ther. 2009;11(2):99-106.

45. Pérez-Ferre N, Galindo M, Fernández MD, et al. A telemedicine system based on internet and short message service as a new approach in the follow-up of patients with gestational diabetes. Diabetes Res Clin Pract. 2010;87(2):e15-e17.

46. Strandbygaard U, Thomsen SF, Backer V. A daily SMS reminder increases adherence to asthma treatment: a three-month follow-up study. Respir Med. 2010;104(2):166-171.

47. Lv Y, Zhao H, Liang Z, et al. A mobile phone short message service improves perceived control of asthma: a randomized controlled trial. Telemed J E Health. 2012;18(6):420-426.

48. Petrie KJ, Perry K, Broadbent E, Weinman J. A text message programme designed to modify patients' illness and treatment beliefs improves self-reported adherence to asthma preventer medication. Br J Health Psychol. 2012;17(1):74-84.

49. Slack MK, Brooks AJ. Medication management issues for adolescents with asthma. Am J Health Syst Pharm. 1995;52(13):1417-1421.

50. Rhee H, Wenzel J, Steeves RH. Adolescents' psychosocial experiences living with asthma: a focus group study. J Pediatr Health Care. 2007;21(2):99-107.

51. Ayala GX, Miller D, Zagami E, Riddle C, Willis S, King D. Asthma in middle schools: what students have to say about their asthma. $J$ Sch Health. 2006;76(6):208-214.

52. Lenhart A, Purcell K, Smith A, Zickuhr K. Social Media and Mobile Internet use among Teens and Young Adults. Washington: Pew Internet and American Life Project; 2010.
Patient Preference and Adherence

\section{Publish your work in this journal}

Patient Preference and Adherence is an international, peer-reviewed, open access journal focusing on the growing importance of patient preference and adherence throughout the therapeutic continuum. Patient satisfaction, acceptability, quality of life, compliance, persistence and their role in developing new therapeutic modalities and compounds to

\section{Dovepress}

optimize clinical outcomes for existing disease states are major areas of interest. This journal has been accepted for indexing on PubMed Central. The manuscript management system is completely online and includes a very quick and fair peer-review system. Visit http://www.dovepress.com/ testimonials.php to read real quotes from published authors. 